\title{
Microbiological Characterization of Salty Bread, Soy Cheese and Three Yogurt Varieties Sold in the Streets of Benin
}

\author{
A. A. M. Djogbe ${ }^{1 *}$, C. K. C. Tchekessi ${ }^{1}$, P. Sachi ${ }^{1}$, C. Degbey ${ }^{2}$, R. Bleoussi ${ }^{1}$, J. Banon ${ }^{1}$, \\ K. Assogba ${ }^{1}$, E. M. Ouendo ${ }^{3}$ and I. Bokossa Yaou ${ }^{1}$
}

${ }^{1}$ Food Safety Research Unit (URSSA), Laboratory of Microbiology and Food Technology (LAMITA), University of Abomey-Calavi, 04 P.O.Box 1107 Cotonou, Republic of Benin

${ }^{2}$ Laboratory of Research and Expertise in Public Health of the National University Hospital Center Hubert Koutoukou Maga, Republic of Benin

${ }^{3}$ Public Health Laboratory of the Regional Institute of Public Health (IRSP), University of Abomey-Calavi, Ouidah, Republic of Benin

*Corresponding author

\section{A B S T R A C T}

Foodstuffs is a favorable environment for microorganism's growth. Thus, the germs likely to be found in these foods can be at the base of several food poisoning. The objective of the study was to evaluate the microbiological quality of some foods prepared and sold in

\section{Keywords}

Toxi-food infections, food insalubrity, salty bread, soy cheese, yogurt, Benin

\section{Article Info}

Accepted:

18 July 2019

Available Online:

10 August 2019 Benin. To do this, a prospective descriptive survey was conducted in five major cities (Cotonou, Abomey-Calavi, Porto-Novo, Lokossa and Abomey) of Benin. It collected samples of salted bread, soy cheese and three yogurt varieties (Dolait, Tropical and Comtesse) for microbiological analyzes. Out of the three varieties of yoghurt, the results of the work revealed that the microbial loads in CFU / g of salty breads and soy cheeses respectively amounted to $21.4810^{3}$ and $25.7310^{3}$ in total flora, $11.5010^{2}$ and $22.2910^{2}$ in total coliforms, $7.4010^{2}$ and $12.6110^{2}$ in thermo-tolerant coliforms, $60.8010^{2}$ and 217.84 $10^{2}$ in staphylococci then $21.4310^{2}$ and $113.2410^{2}$ in yeast were not in accordance with the values required by the criteria of Standard No. 2073/2005. The identification of isolated organisms showed that salty breads and soy cheeses contained the bacteria of interest in toxi-food infections such as Acinetobacter calcoaceticus, Staphylococcus aureus, Salmonella typhi, Shigella sp., Citrobacter fameri, Klebsiella pneumoniae, Enterobacter cloacae, Raoultella ornithinolytica, Escherichia coli and Enterobacter aerogenes. This shows that these foods require better health surveillance for the well-being of the populations.

\section{Introduction}

Food is of paramount importance in the life of man. To satisfy his needs, man feeds on different categories of food: meat, fish or eggs, dairy products, fats, vegetables and fruits, cereals and legumes, sugars. Each of them has a specific role in the proper functioning of the body. There are, however, several food-related hazards that can be detrimental to human health, although essential. Infectious diseases of food origin 
represent a significant burden in the world. Every year, millions of people around the world suffer from food poisoning of all kinds; almost one in ten falls ill from this cause (WHO, 2015a). They can be fatal especially in children under five years of age with a $33 \%$ of deaths (WHO, 2015b). In Africa, especially, food-borne diseases cause more than 91 million patients, of whom 137,000 die, which represents $1 / 3$ of deaths from global mortality due to these diseases (WHO, 2015b). Infections transmitted to humans by food are a real problem of international health. They persist in industrialized countries as well as in developing countries, emerging or in health and economic transition (Kaferstein and Abdussalam, 1999; Malvy et al., 2003). The uncontrolled application of chemicals in agriculture, environmental contamination, the use of unauthorized additives, microbiological hazards, or other abuses of food throughout the food chain can contribute to introducing hazards directly related to food or preventing them from being reduced (FAO, 2001). The socio-economic situation, the rapid urbanization of the developing countries and many other factors (poverty, etc.) have facilitated the emergence of new modes of consumption in the informal sector: these are "street foods". They define themselves as ready-to-eat foods, prepared and sold by vendors or peddlers, especially on streets and public places (Baba-Moussa et al., 2006). These street foods are not always prepared., kept and sold under good hygienic conditions There are three main categories of street food in Africa: ready meals, snacks and beverages, most of which are made from local products (cereals, tubers, legumes, fruits and vegetables, meat products) using traditional technologies that are rarely improved (Houssou et al., 2015; Michaud and Vodouhè, 2012). In Benin, many cases of toxi-food infections have reported (Allogni et al., 2010; Badarou and Coppieters, 2009; Fayomi B., 1992), according to (Ahoyo et al., 2010;
Chauliac et al., 1998), their frequency is largely underestimated by the authorities. Their origins are rarely determined by the weakness of diagnostic means, including microbiological means (Fayomi B., 1992). Toxi-food infections is manifested as major symptoms: digestive diseases such as diarrhea, vomiting, nausea, abdominal cramps, constipation, etc. (Baba-Moussa et al., 2006); but also clinical signs such as excessive thirst, excessive salivation, fever, chills (Belomaria and Khadmaoui, 2017). A food-borne illness is defined as the appearance in one or two grouped cases, of a similar symptomatology, most often of the gastrointestinal type whose cause may relate to the same food origin. It generally results from two consecutive mechanisms: the contamination by bacteria of a product intended for consumption and proliferation of these bacteria resulting in the development of a toxin or the constitution of an infectious inoculum. Multiple microorganisms (bacteria, viruses, parasites) are likely to contaminate foodstuffs and cause various pathologies (Haour, 2018; Tanouti, 2016). The growing awareness of the adverse health effects of toxi-food infections, the importance of global food trade and the requirement of healthy food for consumers are such that the risk analysis associated with food has acquired unprecedented importance (FAO, 2001). The present study proposes to evaluate the microbiological quality of some staple foods in Benin: bread, soy cheese (tofu) and yoghurt.

\section{Materials and Methods}

\section{Materials}

The field equipment consisted mainly of stomacher ND bags, a marker and a cooler containing cold accumulators for the preservation of samples. Salty bread, soy cheese and yoghurt (3 varieties of their trade name "Dolait", "Tropical" and "Comtesse") 
constituted the biological material. The analytical material used was the standard microbiological laboratory equipment.

\section{Methods}

\section{Descriptive prospective study}

This prospective descriptive study was conducted in Cotonou, Abomey-Calavi, PortoNovo, Lokossa, and Abomey communes over a 9-month period from April to December 2018.

A total of 576 samples were collected including 288 breads (144 morning breads and 144 evening bread) and 288 soy cheeses (144 morning soy and 144 evening soy cheeses). These sizes were determined by the Dagnelie formula (1998): $\mathrm{n}=4 \mathrm{p}(1-\mathrm{p}) / \mathrm{d} 2$; where $\mathrm{n}$ is the sample size, with a margin of error of 0.05 and $\mathrm{p}$, the prevalence of foodborne diseases (WHO, 2015a) is $10 \%$. The distribution by commune was made on the basis of the RGPH-4, 2013 (INSAE, 2013).

The three varieties of yogurt were sampled only in the city of Abomey-Calavi because it is a product manufactured by the same company and distributed throughout the territory.

The choice of sellers was random. The food (salt bread, soy cheese and yoghurt) is bought and put in stomacher ${ }^{\mathrm{ND}}$ bags. It is then labeled and placed in a cooler containing cold accumulators in order to be delivered to the laboratory under good storage conditions for analysis.

\section{Microbiological quality assessment}

The microbiological analyzes consisted in counting total mesophilic flora (ISO 4833, 2003) on PCA - HIMEDIA M091 (Plate Count Agar), yeast and mold (NF ISO 21527-
2, 2008) on Sabouraud Dextrose Agar (Oxoid CM 0041).) with chloramphenicol (0.05g / 1), total coliforms (NF ISO 4832 (V 08-015), 2006), and thermo-tolerant coliforms (NF ISO 4832 (V08-060), 2009) on VRBA - Oxoid CM 0485 (Violet Red Bile Agar), Staphylococcus aureus (NF EN ISO 6888-1/A1 (V 08-0141/A1), 2004) on Baird-Parker Agar (BP OXOID CM0275) with egg yolk and potassium tellurite, Anaerobic sulphitereducing bacteria (NF V08-061, 2009) on TSN Agar and Salmonella (ISO 6579, 2002) on SS Agar. Enumeration was done by counting colonies (Guiraud and Galzy, 1980). These microbiological analyzes were performed in triplicate on each product sample.

Identification of interest germs in the toxifood infections

Salmonella research and the identification of certain of interest germs in toxi-food infections were also carried out thanks to Biomérieux API 20E gallery and Thermo Fisher Scientific RapID One System REMEL gallery.

\section{Statistical analyzes of the data}

All data collected from analysis were processed using MINITAB 16.0 software that permitted to make analysis of variance (ANOVA) and Tukey's test for comparison of means. The significance level of 5\% is selected $(\mathrm{p}<0.05)$.

\section{Results and Discussion}

\section{Microbiological assessment characteristics of salty bread}

In Table 1 are presented the results of microbiological analyzes of salt bread sampled in the cities of Cotonou, AbomeyCalavi, Porto-Novo, Lokossa and Abomey. 
A significant difference was observed between morning and evening bread sample data for total mesophilic aerobic flora, total coliforms, heat-tolerant, yeast, and staphylococci.

The samples of Lokossa bread (morning: $11.1610^{3} \mathrm{CFU} / \mathrm{g}$ and evening: $11.4810^{3}$ CFU / g) were the least soiled and those of Abomey-Calavi (morning: $14.9010^{3} \mathrm{CFU} / \mathrm{g}$ and evening: $21.4810^{3} \mathrm{CFU} / \mathrm{g}$ ) the most contaminated.

The total coliform microbial loads for the morning bread samples reached a value of $6.8210^{2} \mathrm{CFU} / \mathrm{g}$ while the evening ones ranged from $1.1010^{2} \mathrm{CFU} / \mathrm{g}$ to $11.5010^{2}$ CFU / g. The morning and evening bread samples from Abomey had the highest microbial load values for total coliforms.

The city of Porto-Novo had bread samples (morning $6.1810^{2} \mathrm{CFU} / \mathrm{g}$ : and evening: 7.40 $10^{2} \mathrm{CFU} / \mathrm{g}$ ) which contained more thermotolerant coliforms while in Cotonou they did not contain any.

$11.0610^{2} \mathrm{CFU} / \mathrm{g}$ and $21.4310^{2} \mathrm{CFU} / \mathrm{g}$ were respectively the maximum values of the microbial yeast loads of morning and evening bread samples. The Porto-Novo bread samples (morning and evening) had developed more yeasts while those from Abomey had not developed any.

For staphylococci, morning bread samples from Abomey (18.98 $10^{2} \mathrm{CFU} / \mathrm{g}$ ) and those from Abomey-Calavi evening $\left(60.8010^{2} \mathrm{CFU}\right.$ / g) had the highest loads.

\section{Evaluation of microbiological characteristics of soy cheeses}

Table 2 shows the results of microbiological analyzes of soy cheese sampled in the cities of Cotonou, Abomey-Calavi, Porto-Novo, Lokossa and Abomey.
A significant difference was also observed between morning and evening soybean cheese samples for microbial loads of total mesophilic aerobic flora, total coliforms, heattolerant, yeasts and staphylococci.

The mean value of the microbial loads of total mesophilic aerobic flora for morning and evening soybean cheese samples was 14.71 $10^{3} \mathrm{CFU} / \mathrm{g}$ and $19.7210^{3} \mathrm{CFU} / \mathrm{g}$, respectively. The Cotonou soy cheeses (morning and evening) were the most soiled.

Total coliforms were present in the morning and evening soybean cheese samples with respective averages of $7.6110^{2} \mathrm{CFU} / \mathrm{g}$ and $12.5210^{2} \mathrm{CFU} / \mathrm{g}$. Porto-Novo soy cheeses had fewer total coliforms than in other cities.

The Lokossa morning soybean cheese samples had few thermo-tolerant coliforms $\left(0.9410^{2}\right.$ CFU / g) while the evening ones had the highest values $\left(12.6110^{2} \mathrm{CFU} / \mathrm{g}\right)$.

The values $\left(13.5210^{2} \mathrm{CFU} / \mathrm{g}\right.$ and $71.8210^{2}$ $\mathrm{CFU} / \mathrm{g}$ ) were the minimum and maximum yeast values, respectively, of the morning soybean cheese samples; $16.8510^{2} \mathrm{CFU} / \mathrm{g}$ and $113.2410^{2}$ corresponded to those of the evening. Abomey (morning and evening) had the highest values.

Abomey's morning soy cheeses had the highest microbial loadings for staphylococci.

\section{Evaluation of the microbiological characteristics of yoghurts}

The results of microbiological analyzes of yogurt sampled are presented in Table 3.

Total mesophilic aerobic flora values of yogurt samples varied between $11810^{3} \mathrm{CFU} /$ $\mathrm{g}$ to $81010^{3} \mathrm{CFU} / \mathrm{g}$; those of total coliforms situated between less than $10 \mathrm{CFU} / \mathrm{g}$ to 30 CFU / g. All yogurts had microbial charges in 
thermo-tolerant coliforms <10 CFU / g. Yeast contents in yogurt ranged from less than 10 $\mathrm{CFU} / \mathrm{g}$ to 1.05 to $10^{2} \mathrm{CFU} / \mathrm{g}$.

As for the molds, their values were in the $<10$ $\mathrm{CFU} / \mathrm{g}$ to $0.5010^{2} \mathrm{CFU} / \mathrm{g}$.

The minimum and maximum microbial load values for staphylococci in the yogurt samples were $0.1010^{2} \mathrm{CFU} / \mathrm{g}$ and $0.3510^{2} \mathrm{CFU} / \mathrm{g}$, respectively.

\section{Identification of interest germs in toxi-food infections}

The identified organisms were mainly Acinetobacter calcoaceticus, Staphylococcus aureus, Salmonella typhi, Shigella sp., Citrobacter fameri, Klebsiella pneumoniae, Raoultella ornithinolytica, Escherichia coli, Enterobacter and aerogenes, Enterobacter cloacae.

Table 4 reveals that Acinetobacter calcoaceticus was identified in the Port-Novo, Abomey and only in the Lokossa Evening bread samples with a predominance in evening-Porto-Novo bread samples (76.19\%) while Table 10 reports its presence in the samples of evening soy cheese from Cotonou $(5.56 \%)$ and Abomey (12.50\%).

Table 5 and 17 indicate that Staphylococcus aureus was present in all bread and soy cheese samples from all cities.

Table 6 indicates that Salmonella typhi was identified only in Abomey bread samples (25\%).

Table 7 informs that Shigella sp. was found in the evening bread samples of Porto-Novo and Abomey (25\%) while in Table 18, Shigella sp. was isolated in all of Lokossa's soybean cheese samples (morning and evening) $(100 \%)$.
From Table 8, it appears that Citrobacter fameri was detected only in the evening bread samples from Abomey (25\%).

In contrast, it was found in all evening soy cheese samples from all cities except PortoNovo (Table 11).

Table 9 shows that Klebsiella pneumoniae was only present in the evening bread samples from Abomey-Calavi (3.77\%). However, in soy cheese samples it was identified in all cities except Lokossa (Table 12).

Table 13 reports that Enterobacter cloacae was not detected in the Porto-Novo and Lokossa soy cheese samples.

Those of Abomey evening had the highest identification rate $(37.50 \%)$.

Raoultella ornithinolytica was present in evening soybean cheese samples from Cotonou, Abomey-Calavi and Porto-Novo with a predominance in Abomey-Calavi (24.53\%) (Table 14).

From Table 15, Escherichia coli was present in samples of evening soybean cheese (7.41\%), Abomey-Calavi (3.77\%) and Abomey (12.50\%).

Table 16 reveals that Enterobacter aerogenes was highly isolated in Abomey-Calavi evening soybean cheese samples (11.32\%).

The results of microbial loads of total mesophilic aerobic flora for bread samples are higher than the standard $\left(10^{4} \mathrm{CFU} / \mathrm{g}\right)$ according to the regulation $n \circ 2073 / 2005$ (Union Européenne, 2005). These high values are due to poor conservation of bread sold in the streets. The maximum values obtained are lower than those $\left(1.2010^{2}-3.7810^{4} \mathrm{CFU} / \mathrm{g}\right)$ of Ennadir et al., (2012) who worked on wheat flour, a raw material for bread production. 
Table.1 Mean values in CFU / $\mathrm{g}$ of germs counted in bread samples taken morning and evening in cities

\begin{tabular}{|c|c|c|c|c|c|}
\hline Sample & FAMT $\left(10^{3}\right)$ & $\begin{array}{c}\text { Total coliforms } \\
\left(10^{2}\right)\end{array}$ & $\begin{array}{c}\text { Thermo- } \\
\text { tolerant } \\
\text { coliforms }\left(\mathbf{1 0}^{\mathbf{2}}\right)\end{array}$ & Yeast $\left(10^{2}\right)$ & $\begin{array}{c}\text { Staphylococcus } \\
\left(10^{2}\right)\end{array}$ \\
\hline \multicolumn{6}{|c|}{ Morming } \\
\hline PAm & $14.79 \pm 0.070^{f}$ & $6.82 \pm 0.132^{\mathrm{h}}$ & $3.84 \pm 0.165^{\mathrm{e}}$ & $0.00 \pm 0.000^{\mathrm{a}}$ & $18.98 \pm 0.030^{g}$ \\
\hline PLm & $11.16 \pm 0.100^{\mathrm{a}}$ & $0.00 \pm 0.000^{\mathrm{a}}$ & $1.71 \pm 0.142^{b}$ & $1.59 \pm 0.160^{\mathrm{b}}$ & $5.68 \pm 0.060^{c}$ \\
\hline PPNm & $12.53 \pm 0.126^{\mathrm{d}}$ & $1.80 \pm 0.100^{\mathrm{d}}$ & $6.18 \pm 0.106^{\mathrm{g}}$ & $11.06 \pm 0.182^{\mathrm{f}}$ & $6.17 \pm 0.110^{d}$ \\
\hline PABm & $14.91 \pm 0.045^{\mathrm{c}}$ & $1.22 \pm 0.000^{\mathrm{b}}$ & $0.10 \pm 0.000^{\mathrm{a}}$ & $4.53 \pm 0.000^{d}$ & $1.37 \pm 0.050^{\mathrm{a}}$ \\
\hline PCotm & $14.46 \pm 0.154^{\mathrm{e}}$ & $1.10 \pm 0.000^{b}$ & $0.00 \pm 0.000^{\mathrm{a}}$ & $0.95 \pm 0.000^{\mathrm{a}}$ & $1.44 \pm 0.019^{b}$ \\
\hline \multicolumn{6}{|c|}{ Evening } \\
\hline PAs & $19.87 \pm 0.097^{j}$ & $11.50 \pm 0.115^{\mathrm{i}}$ & $4.06 \pm 0.099^{f}$ & $0.00 \pm 0.000^{\mathrm{a}}$ & $28.41 \pm 0.043^{\mathrm{h}}$ \\
\hline PLs & $11.48 \pm 0.126^{\mathrm{b}}$ & $1.48 \pm 0.040^{c}$ & $3.02 \pm 0.120^{\mathrm{d}}$ & $4.89 \pm 0.154^{\mathrm{e}}$ & $7.78 \pm 0.075^{\mathrm{t}}$ \\
\hline PPNs & $17.38 \pm 0.120^{\mathrm{i}}$ & $6.06 \pm 0.089^{\mathrm{g}}$ & $7.40 \pm 0.094^{\mathrm{h}}$ & $21.43 \pm 0.175^{\mathrm{g}}$ & $6.88 \pm 0.105^{\mathrm{e}}$ \\
\hline PABs & $21.48 \pm 0.078^{\mathrm{g}}$ & $4.72 \pm 0.084^{\mathrm{t}}$ & $2.26 \pm 0.121^{\mathrm{c}}$ & $29.32 \pm 0.063^{\mathrm{h}}$ & $60.80 \pm 0.075^{1}$ \\
\hline PCots & $17.27 \pm 0.110^{\mathrm{h}}$ & $2.42 \pm 0.115^{\mathrm{e}}$ & $0.00 \pm 0.000^{\mathrm{a}}$ & $2.78 \pm 0.045^{\mathrm{c}}$ & $7.79 \pm 0.026^{\mathrm{f}}$ \\
\hline ard & $10^{4}$ & & - & $510^{2}$ & \\
\hline
\end{tabular}

Legend: PA: Abomey bread; PL: Lokossa bread; PPN: Porto Novo bread; PAB: Abomey-Calavi bread; PCot: Cotonou bread; m: Morning; s: Evening; FAMT: Total mesophilic aerobic flora.

Means with the same letters in the same column are not significantly different $(\mathrm{p}<0.05)$. The data represented in this table are the averages of three repetitions ( \pm deviation).

Standards: Regulation (EC) N N $^{\circ}$ 2073/2005

Table.1 Mean values in CFU / $g$ of germs counted in morning and evening samples of soybeans in cities

\begin{tabular}{|c|c|c|c|c|c|}
\hline Sample & FAMT ${\left(\mathbf{1 0}^{\mathbf{3}}\right)}^{\left.\text {Total coliforms } \mathbf{( 1 0}^{\mathbf{2}}\right)}$ & $\begin{array}{c}\text { Thermo-tolerant } \\
\left.\text { Coliforms } \mathbf{( 1 0}^{\mathbf{2}}\right)\end{array}$ & Yeast $\left.\mathbf{( 1 0}^{\mathbf{2}}\right)$ & $\begin{array}{c}\text { Staphylococcus } \\
\left(\mathbf{1 0}^{\mathbf{2}}\right)\end{array}$ \\
\hline \multicolumn{7}{|c|}{ Morning } \\
\hline SAm & $15.00 \pm 0.054^{\mathrm{c}}$ & $14.21 \pm 0.100^{\mathrm{i}}$ & $1.68 \pm 0.120^{\mathrm{b}}$ & $71.82 \pm 0.108^{\mathrm{g}}$ & $182.39 \pm 0.042^{\mathrm{i}}$ \\
\hline SLm & $14.84 \pm 0.104^{\mathrm{b}}$ & $2.08 \pm 0.143^{\mathrm{b}}$ & $0.94 \pm 0.132^{\mathrm{a}}$ & $13.52 \pm 0.100^{\mathrm{a}}$ & $26.65 \pm 0.099^{\mathrm{c}}$ \\
\hline SPNm & $14.75 \pm 0.032^{\mathrm{b}}$ & $1.94 \pm 0.090^{\mathrm{a}}$ & $4.04 \pm 0.067^{\mathrm{e}}$ & $13.92 \pm 0.138^{\mathrm{b}}$ & $4.65 \pm 0.027^{\mathrm{a}}$ \\
\hline SABm & $13.53 \pm 0.120^{\mathrm{a}}$ & $8.16 \pm 0.054^{\mathrm{d}}$ & $5.78 \pm 0.040^{\mathrm{f}}$ & $57.46 \pm 0.113^{\mathrm{f}}$ & $97.78 \pm 0.078^{\mathrm{d}}$ \\
\hline SCotm & $15.40 \pm 0.105^{\mathrm{e}}$ & $11.65 \pm 0.065^{\mathrm{f}}$ & $3.59 \pm 0.161^{\mathrm{d}}$ & $48.02 \pm 0.030^{\mathrm{e}}$ & $129.51 \pm 0.050^{\mathrm{g}}$ \\
\hline \multicolumn{7}{|c|}{ Evening } \\
\hline SAs & $18.23 \pm 0.070^{\mathrm{g}}$ & $12.54 \pm 0.089^{\mathrm{g}}$ & $2.86 \pm 0.150^{\mathrm{c}}$ & $113.24 \pm 0.127^{\mathrm{j}}$ & $217.84 \pm 0.055^{\mathrm{j}}$ \\
\hline SLs & $15.14 \pm 0.011^{\mathrm{d}}$ & $10.11 \pm 0.152^{\mathrm{e}}$ & $12.61 \pm 0.106^{\mathrm{j}}$ & $26.65 \pm 0.096^{\mathrm{d}}$ & $114.15 \pm 0.074^{\mathrm{f}}$ \\
\hline SPNs & $17.44 \pm 0.039^{\mathrm{f}}$ & $4.99 \pm 0.075^{\mathrm{c}}$ & $9.41 \pm 0.034^{\mathrm{g}}$ & $16.85 \pm 0.120^{\mathrm{c}}$ & $6.95 \pm 0.044^{\mathrm{b}}$ \\
\hline SABs & $22.04 \pm 0.116^{\mathrm{h}}$ & $12.69 \pm 0.010^{\mathrm{h}}$ & $10.65 \pm 0.082^{\mathrm{h}}$ & $86.77 \pm 0.103^{\mathrm{i}}$ & $100.06 \pm 0.080^{\mathrm{e}}$ \\
\hline SCots & $25.73 \pm 0.096^{\mathrm{i}}$ & $22.29 \pm 0.078^{\mathrm{j}}$ & $11.16 \pm 0.123^{\mathrm{i}}$ & $74.54 \pm 0.042^{\mathrm{h}}$ & $146.05 \pm 0.045^{\mathrm{h}}$ \\
\hline Standard & - & - & - & - & $10^{2}-10^{3}$ \\
\hline
\end{tabular}

Legend: SA: Abomey soy cheese ; SL: Lokossa soy cheese ; SPN: Porto Novo soy cheese ; SAB: AbomeyCalavi soy cheese ; SCot: Cotonou soy cheese ; m: Morning ; s : Evening ; FAMT : Total mesophilic aerobic flora. Means with the same letters in the same column are not significantly different $(\mathrm{p}<0.05)$. The data represented in this table are the averages of three repetitions ( \pm deviation).

Standards: Regulation (EC) $\mathrm{N}^{\circ} .2073 / 2005$. 
Table.2 Mean values in CFU / g of germs counted in Yogurt samples

\begin{tabular}{|c|c|c|c|c|c|c|c|c|}
\hline Sample & FAMT $\left(10^{3}\right)$ & $\begin{array}{l}\text { Total coliforms } \\
\left(\mathbf{1 0}^{2}\right)\end{array}$ & $\begin{array}{c}\text { Thermo- } \\
\text { tolerant } \\
\text { coliforms }\left(\mathbf{1 0}^{2}\right)\end{array}$ & Yeast $\left(10^{2}\right)$ & Molds $\left(\mathbf{1 0}^{2}\right)$ & $\begin{array}{c}\text { Staphylococcus } \\
\left(\mathbf{1 0}^{2}\right)\end{array}$ & $\begin{array}{l}\text { Sulphito-reducing } \\
\text { anaerobic bacteria }\end{array}$ & Salmonella \\
\hline Ytrop & $810.00 \pm 0.028^{c}$ & $0.30 \pm 0.000^{\mathrm{a}}$ & $<10^{\mathrm{a}}$ & $1.05 \pm 0.050^{b}$ & $0.50 \pm 0.020^{b}$ & $0.35 \pm 0.030^{c}$ & Absent & Absent \\
\hline Ycomt & $430.00 \pm 0.026^{b}$ & $<10^{\mathrm{a}}$ & $<10^{\mathrm{a}}$ & $<10^{\mathrm{a}}$ & $<10^{\mathrm{a}}$ & $010 \pm 0.000^{\mathrm{a}}$ & Absent & Absent \\
\hline Ydlt & $118.00 \pm 0.020^{\mathrm{a}}$ & $0.10 \pm 0.000^{\mathrm{a}}$ & $<10^{\mathrm{a}}$ & $<10^{\mathrm{a}}$ & $0.50 \pm 0.010^{b}$ & $0.20 \pm 0.019^{b}$ & Absent & Absent \\
\hline Standard & - & $10-10^{2}$ & $1-10$ & $10^{2}-10^{3}$ & Absent & $10-10^{2}$ & - & Absent $/ 25 \mathrm{~g}$ \\
\hline
\end{tabular}

Legend: Ytrop : Tropical Yogurt; Ycomt : Comtesse Yogurt ; Ydlt : Dolait Yogurt; FAMT : Total mesophilic aerobic flora

Means with the same letters in the same column are not significantly different $(\mathrm{p}<0.05)$. The data represented in this table are the averages of three repetitions $( \pm$ deviation).

Standards: JORA : 035 of 27-05-1998 
Table.3 Distribution of Acinetobacter calcoaceticus in bread samples (morning and evening) by city

\begin{tabular}{|c|c|c|c|c|}
\hline Cities & $\begin{array}{c}\text { Numbers Acinetobacter } \\
\text { calcoaceticus } \\
\text { (morning) }\end{array}$ & $\%$ & $\begin{array}{c}\text { Numbers Acinetobacter } \\
\text { calcoaceticus } \\
\text { (Evening) }\end{array}$ & $\%$ \\
\hline Cotonou & 0 & 0.00 & 0 & 0.00 \\
\hline Abomey-Calavi & 0 & 0.00 & 0 & 0.00 \\
\hline Porto-Novo & 9 & 42.86 & 16 & 76.19 \\
\hline Lokossa & 0 & 0.00 & 2 & 25.00 \\
\hline Abomey & 4 & 50.00 & 6 & 75.00 \\
\hline
\end{tabular}

Table.4 Distribution of Staphylococcus aureus in bread samples (morning and evening) by city

\begin{tabular}{|c|c|c|c|c|}
\hline Cities & $\begin{array}{c}\text { Numbers Staphylococcus } \\
\text { aureus (morning) }\end{array}$ & $\%$ & $\begin{array}{c}\text { Numbers } \\
\text { Staphylococcus aureus } \\
\text { (evening) }\end{array}$ & $\%$ \\
\hline Cotonou & 4 & 7.41 & 8 & 14.81 \\
\hline Abomey-Calavi & 11 & 20.75 & 31 & 58.49 \\
\hline Porto-Novo & 11 & 52.38 & 12 & 57.14 \\
\hline Lokossa & 4 & 50.00 & 6 & 75.00 \\
\hline Abomey & 1 & 12.50 & 2 & 25.00 \\
\hline
\end{tabular}

Table.5 Distribution of Salmonella typhi in bread samples (morning and evening) by city

\begin{tabular}{|c|c|c|c|c|}
\hline Cities & $\begin{array}{c}\text { Numbers Salmonella } \\
\text { typhi (morning) }\end{array}$ & $\boldsymbol{\%}$ & $\begin{array}{c}\text { Numbers Salmonella } \\
\text { typhi (evening) }\end{array}$ & $\%$ \\
\hline Cotonou & 0 & 0.00 & 0 & 0.00 \\
\hline Abomey-Calavi & 0 & 0.00 & 0 & 0.00 \\
\hline Porto-Novo & 0 & 0.00 & 0 & 0.00 \\
\hline Lokossa & 0 & 0.00 & 0 & 0.00 \\
\hline Abomey & 2 & 25.00 & 2 & 25.00 \\
\hline
\end{tabular}

Table.6 Distribution of Shigella sp. in bread samples (morning and evening) by city

\begin{tabular}{|c|c|c|c|c|}
\hline Cities & $\begin{array}{c}\text { Numbers Shigella } \boldsymbol{s p} \\
\text { (morning) }\end{array}$ & $\boldsymbol{\%}$ & $\begin{array}{c}\text { Numbers Shigella sp } \\
\text { (soir) }\end{array}$ & $\%$ \\
\hline Cotonou & 0 & 0.00 & 0 & 0.00 \\
\hline Abomey-Calavi & 0 & 0.00 & 0 & 0.00 \\
\hline Porto-Novo & 0 & 0.00 & 2 & 9.52 \\
\hline Lokossa & 0 & 0.00 & 6 & 75.00 \\
\hline Abomey & 1 & 12.25 & 2 & 25.00 \\
\hline
\end{tabular}


Table.7 Distribution of Citrobacter fameri in bread samples (morning and evening) by city

\begin{tabular}{|c|c|c|c|c|}
\hline Cities & $\begin{array}{c}\text { Numbers Citrobacter } \\
\text { fameri (morning) }\end{array}$ & $\%$ & $\begin{array}{c}\text { Numbers Citrobacter } \\
\text { fameri (evening) }\end{array}$ & $\%$ \\
\hline Cotonou & 0 & 0.00 & 0 & 0.00 \\
\hline Abomey-Calavi & 0 & 0.00 & 0 & 0.00 \\
\hline Porto-Novo & 0 & 0.00 & 0 & 0.00 \\
\hline Lokossa & 0 & 0.00 & 0 & 0.00 \\
\hline Abomey & 0 & 0.00 & 2 & 25.00 \\
\hline
\end{tabular}

Table.8 Distribution of Klebsiella pneumoniae in bread samples (morning and evening) by city

\begin{tabular}{|c|c|c|c|c|}
\hline Cities & $\begin{array}{c}\text { Numbers Klebsiella } \\
\text { pneumoniae (morning) }\end{array}$ & $\%$ & $\begin{array}{c}\text { Numbers Klebsiella } \\
\text { pneumoniae (evening) }\end{array}$ & $\%$ \\
\hline Cotonou & 0 & 0.00 & 0 & 0.00 \\
\hline Abomey-Calavi & 0 & 0.00 & 2 & 3.77 \\
\hline Porto-Novo & 0 & 0.00 & 0 & 0.00 \\
\hline Lokossa & 0 & 0.00 & 0 & 0.00 \\
\hline Abomey & 0 & 0.00 & 0 & 0.00 \\
\hline
\end{tabular}

Table.9 Distribution of Acinetobacter calcoaceticus in soybean cheese samples (morning and evening) by city

\begin{tabular}{|c|c|c|c|c|}
\hline Cities & $\begin{array}{l}\text { Numbers Acinetobacter } \\
\text { calcoaceticus (morning) }\end{array}$ & $\%$ & $\begin{array}{c}\text { Numbers Acinetobacter } \\
\text { calcoaceticus (evening) }\end{array}$ & $\%$ \\
\hline Cotonou & 0 & 0.00 & 3 & 5.56 \\
\hline Abomey-Calavi & 0 & 0.00 & 0 & 0.00 \\
\hline Porto-Novo & 0 & 0.00 & 0 & 0.00 \\
\hline Lokossa & 0 & 0.00 & 0 & 0.00 \\
\hline Abomey & 0 & 0.00 & 1 & 12.50 \\
\hline
\end{tabular}

Table.10 Distribution of Citrobacter fameri in soybean cheese samples (morning and evening) by city

\begin{tabular}{|c|c|c|c|c|}
\hline Cities & $\begin{array}{c}\text { Numbers Citrobacter } \\
\text { fameri (morning) }\end{array}$ & $\%$ & $\begin{array}{c}\text { Numbers Citrobacter } \\
\text { fameri (evening) }\end{array}$ & $\%$ \\
\hline Cotonou & 0 & 0.00 & 4 & 7.41 \\
\hline Abomey-Calavi & 2 & 3.77 & 2 & 3.77 \\
\hline Porto-Novo & 0 & 0.00 & 0 & 0.00 \\
\hline Lokossa & 0 & 0.00 & 2 & 25.00 \\
\hline Abomey & 0 & 0.00 & 1 & 12.50 \\
\hline
\end{tabular}


Table.11 Distribution of Klebsiella pneumoniae in soybean cheese samples (morning and evening) by city

\begin{tabular}{|c|c|c|c|c|}
\hline Cities & $\begin{array}{c}\text { Numbers Klebsiella } \\
\text { pneumoniae (morning) }\end{array}$ & $\%$ & $\begin{array}{c}\text { Numbers Klebsiella } \\
\text { pneumoniae (evening) }\end{array}$ & $\%$ \\
\hline Cotonou & 7 & 12.96 & 12 & 22.22 \\
\hline Abomey-Calavi & 0 & 0.00 & 7 & 13.21 \\
\hline Porto-Novo & 3 & 14.28 & 3 & 14.28 \\
\hline Lokossa & 0 & 0.00 & 0 & 0.00 \\
\hline Abomey & 2 & 25.00 & 4 & 50.00 \\
\hline
\end{tabular}

Table.12 Distribution of Enterobacter cloacae in soybean cheese samples (morning and evening) by city

\begin{tabular}{|c|c|c|c|c|}
\hline Cities & $\begin{array}{c}\text { Numbers Enterobacter } \\
\text { cloacae (morning) }\end{array}$ & $\%$ & $\begin{array}{c}\text { Numbers Enterobacter } \\
\text { cloacae (evening) }\end{array}$ & $\%$ \\
\hline Cotonou & 6 & 11.11 & 9 & 16.67 \\
\hline Abomey-Calavi & 0 & 0.00 & 2 & 3.77 \\
\hline Porto-Novo & 0 & 0.00 & 0 & 0.00 \\
\hline Lokossa & 0 & 0.00 & 0 & 0.00 \\
\hline Abomey & 2 & 25.00 & 3 & 37.50 \\
\hline
\end{tabular}

Table.13 Distribution of Raoultella ornithinolytica in soybean cheese samples (morning and evening) by city

\begin{tabular}{|c|c|c|c|c|}
\hline Cities & $\begin{array}{c}\text { Numbers Raoultella } \\
\text { ornithinolytica (morning) }\end{array}$ & $\%$ & $\begin{array}{c}\text { Numbers Raoultella } \\
\text { ornithinolytica (evening) }\end{array}$ & $\%$ \\
\hline Cotonou & 0 & 0.00 & 2 & 3.70 \\
\hline Abomey-Calavi & 0 & 0.00 & 13 & 24.53 \\
\hline Porto-Novo & 0 & 0.00 & 2 & 9.52 \\
\hline Lokossa & 0 & 0.00 & 0 & 0.00 \\
\hline Abomey & 0 & 0.00 & 0 & 0.00 \\
\hline
\end{tabular}

Table.14 Distribution of Esherichia coli in soybean cheese samples (morning and evening) by city

\begin{tabular}{|c|c|c|c|c|}
\hline Cities & $\begin{array}{c}\text { Numbers Escherichia } \\
\text { coli (morning) }\end{array}$ & $\%$ & $\begin{array}{c}\text { Numbers Escherichia coli } \\
\text { (evening) }\end{array}$ & $\%$ \\
\hline Cotonou & 0 & 0.00 & 4 & 741 \\
\hline Abomey-Calavi & 0 & 0.00 & 2 & 3.77 \\
\hline Porto-Novo & 0 & 0.00 & 0 & 0.00 \\
\hline Lokossa & 0 & 0.00 & 0 & 0.00 \\
\hline Abomey & 0 & 0.00 & 1 & 12.50 \\
\hline
\end{tabular}


Table.15 Distribution of Enterobacter aerogenes in soybean cheese samples (morning and evening) by city

\begin{tabular}{|c|c|c|c|c|}
\hline Cities & $\begin{array}{c}\text { Numbers Enterobacter } \\
\text { aerogenes (matin) }\end{array}$ & $\boldsymbol{\%}$ & $\begin{array}{c}\text { Numbers Enterobacter } \\
\text { aerogenes (soir) }\end{array}$ & $\%$ \\
\hline Cotonou & 0 & 0.00 & 1 & 1.85 \\
\hline Abomey-Calavi & 0 & 0.00 & 6 & 11.32 \\
\hline Porto-Novo & 0 & 0.00 & 1 & 4.76 \\
\hline Lokossa & 0 & 0.00 & 0 & 0.00 \\
\hline Abomey & 0 & 0.00 & 0 & 0.00 \\
\hline
\end{tabular}

Table.16 Distribution of Staphylococcus aureus in soybean cheese samples (morning and evening) by city

\begin{tabular}{|c|c|c|c|c|}
\hline Cities & $\begin{array}{c}\text { Numbers Staphylococcus } \\
\text { aureus (morning) }\end{array}$ & $\boldsymbol{\%}$ & $\begin{array}{c}\text { Numbers Staphylococcus } \\
\text { aureus (evening) }\end{array}$ & $\%$ \\
\hline Cotonou & 54 & 100 & 54 & 100 \\
\hline Abomey-Calavi & 53 & 100 & 53 & 100 \\
\hline Porto-Novo & 21 & 100 & 21 & 100 \\
\hline Lokossa & 8 & 100 & 8 & 100 \\
\hline Abomey & 8 & 100 & 8 & 100 \\
\hline
\end{tabular}

Table.17 Distribution of Shigella sp. in soybean cheese samples (morning and evening) by city

\begin{tabular}{|c|c|c|c|c|}
\hline Cities & $\begin{array}{c}\text { Numbers Shigella sp } \\
\text { (matin) }\end{array}$ & $\%$ & $\begin{array}{c}\text { Numbers Shigella sp } \\
\text { (soir) }\end{array}$ & $\boldsymbol{\%}$ \\
\hline Cotonou & 36 & 66.67 & 36 & 66.67 \\
\hline Abomey-Calavi & 22 & 41.51 & 22 & 41.51 \\
\hline Porto-Novo & 9 & 42.86 & 9 & 42.86 \\
\hline Lokossa & 8 & 100 & 8 & 100 \\
\hline Abomey & 4 & 50 & 4 & 50 \\
\hline
\end{tabular}

Bread and soy cheese are often placed in baskets covered with thin, transparent fabrics or in transparent glass cases; they are therefore exposed to dust and all kinds of microorganisms in the environment, or to manual handling that is not always hygienic. As pointed out (Barro et al., 2005), street foods, by definition, are very close to the environment, which threatens their hygienic quality at all times. The presence of total and thermo-tolerant coliforms in the bread and soy cheese samples indicates the noncompliance of the sellers with the hygiene rules because the coliforms come from faecal contamination. This is explained by the fact that the sales sites (mainly around the tracks) do not have toilets. Vendors relieve themselves in the open air and do not wash their hands after their needs. The presence of flies (which are generally fecal) and animals around outlets also justify the presence of coliforms. The maximum values of microbial loads in total and heat-tolerant coliforms in bread samples are well above the average values obtained by Ennadir et al., (2012). According to the regulation $\mathrm{n}^{\circ} 2073 / 2005$, 
the microbial loads in yeast of the bread samples are higher than the norm $\left(510^{2}\right.$ UFC / g). These high levels of yeast are the cause of the use of baker's yeast in bread making. Yeast plays a very important role in knowing that it favors the alcoholic fermentation during which the yeasts degrade the sugars contained in wheat flour. This degradation leads to the release of the carbon dioxide $\left(\mathrm{CO}_{2}\right)$ responsible for the lifting of the dough. Apart from some mild gastrointestinal disorders during a massive absorption, yeasts are generally harmless to humans. Their accidental proliferation in foods rich in sugars can, however, cause serious deterioration of these foods. The commercial value of the food is totally lost even if the alteration is superficial, because nobody will buy a degraded product (Tanouti, 2016). The main yeast involved in alcoholic fermentation for the emergence of bread dough is Saccharomyces cerevisiae (Coulibaly et al., 2014; Nguyen, 2015; Rousseaux et al., 2017). It is an occasional commensal yeast of the digestive tract but on a field of immunodeficiency, can be incriminated in various deep mycoses, including urinary tract infections (Saoud et al., 2017).

Soybean is a food derived from soybean processing through soaking, grinding, heating, coagulating, seasoning, cutting and cooking (FAO, 2013). The presence of yeasts in soymilk can be explained by the addition of "guissin" (fermented water from the spontaneous fermentation of maize flour) at the stage of coagulation of soymilk during manufacturing. Indeed, this water comes from the alcoholic fermentation involving yeasts (FAO, 2013).

The microbial loads of staphylococci in soy cheese samples according to Regulation $\mathrm{N}^{\mathrm{o}}$. $2073 / 2005$ are above the standard $\left(10^{2}-10^{3}\right.$ CFU / g). The existence of staphylococci in bread and soybeans indicates a health risk for the consumer. Staphylococci are ubiquitous, non-sporulating, facultative aero-anaerobic organisms, the reservoir of which is localized to the commensal flora of the skin and mucous membranes (nasal, mouth, throat) of warm-blooded animals, and particularly to man. The presence of Staphylococcus aureus, a pathogen, in particular, in foods prepared and handled after cooking is rather a sign of human contamination (lack of hygiene). It may also indicate recontamination with raw materials or poor storage conditions (Centre québécois and Barthe, 2009).

The significant difference $(\mathrm{p}<0.05)$ between the microbial load values of all germs between morning and evening soybean bread and soy cheese samples in addition to the increase in microbial load values of all germs of soy cheese samples and evening bread compared to those of the morning mostly confirms poor preservation and distribution (sale) of these foods. The location of outlets, lack of toilets, unused drinking water, and exhaust pollution, handling of cash at the time of sale, use of mobile phone during the sale, clothing behavioral dirtiness of the sellers are all causes justifying the insalubrity of the food.

Microbiological analysis of the three varieties of yoghurt reveals the absence of pathogenic germs. This is explained not only by compliance with the rules of good production practice but also by the fact that the product being packaged and well preserved is less exposed to the risk of microbial contamination.

The identification of the bacteria such as Acinetobacter calcoaceticus, Staphylococcus aureus, Salmonella typhi, Shigella sp, Citrobacter fameri, Klebsiella pneumoniae, Enterobacter cloacae, Raoultella ornithinolytica, Escherichia coli and Enterobacter aerogenes in salty breads and 
soybean cheeses from this study shows that the targeted foods, outside yoghurts, indicate a high level of contamination by pathogenic microorganisms that cause toxi-food infections. The analyzed foods are therefore not of good hygienic quality. These results are consistent with those of Umba et al., (2018) who identified the same bacteria in breads sold in Kinshasa (Democratic Republic of Congo). Umba et al., (2018) also confirms that this contamination increases with the duration of bread exposure. The risks of occurrence of toxi-food infections in Benin are then not only related to endosulfan in the health zone of Tchaourou (Badarou and Coppieters, 2009); consumer foods sold in private or shared catering (Ahoyo et al., 2010; Baba-Moussa et al., 2006; Barro et al., 2005; Fayomi, 1992; Sossa-Minou et al., 2018); to aflatoxicosis (Allogni et al., 2010); thirstquenching drinks such as fruit juices produced, sold and consumed (Tingbe et al., 2018); meat prepared and sold (Bankole et al., 2012) and many others. This study adds to this long list salt bread and soy cheese. Regardless of the usual bacteria incriminated in toxi-food infections episodes, Acinetobacter calcoaceticus, Citrobacter fameri and Klebsiella pneumoniae are isolated in salty bread and soy cheese while Enterobacter cloacae, Enterobacter aerogenes and Raoultella ornithinolytica are isolated in soy cheese only.

All these species are often found in the environment, in soil, water, wastewater, plants, and animals. They are usually commensal organisms in humans present in the digestive tract. But they are able to switch from commensal to opportunistic pathogen by occasionally causing infections, mainly in vulnerable people with weakened immune systems in hospitals. They are opportunistic pathogens responsible for nosocomial infections that can cause urinary tract infections, pneumonia, abdominal sepsis and brain abscesses, bacteraemia, secondary meningitis and infect wounds (Guérin, 2015; Monsel et al., 2016; WHO, 2004). Direct transmission between human's remains, however, their most common mode of transmission; which may justify their presence in the analyzed foods. In Canada, an outbreak of Citrobacter infections has been associated with the consumption of parsley contaminated with swine manure; eight urinary tract infections and one death were observed (Diallo, 2010). A study in Flagstaff, Arizona indicates that Klebsiella pneumoniae can be a significant foodborne pathogen (Davis et al., 2015).

Soy breads and cheeses sold in the cities of Cotonou, Abomey-Calavi, Porto-Novo, Lokossa and Abomey in view of the microbial load values of the flora of hygienic interest, CT, CTT and yeasts are of quality unsatisfactory according to standard 2073/2005. In addition, the isolated microorganisms Acinetobacter calcoaceticus, Staphylococcus aureus, Salmonella typhi, Shigella sp., Citrobacter fameri, Klebsiella pneumoniae, Enterobacter cloacae, Raoultella ornithinolytica, Escherichia coli and Enterobacter aerogenes are all indicators of food insalubrity that may lead to severe or even lethal poisoning. However, the microbiological quality of the Comtesse yoghurt is satisfactory and that of Dolait and Tropicale yogurts is acceptable.

\section{References}

Ahoyo, T., Ahissou, H., Kounon, F., Aminou, T. \& Dramane, K., 2010. Etude de la qualité bactériologique des aliments vendus sur le campus de l'université d'abomey calavi au bénin. International Journal of Biological and Chemical Sciences, 4.

Allogni, W., Coulibaly, O., Hell, K. \& Mensah, G., 2010. Liens entre la 
contamination des produits maïsicoles à l'aflatoxine et la pauvreté au bénin. Bullettin de la Recherche Agronomique du Bénin, 68, 40-49.

Baba-Moussa, L., Bokossa, Y., Baba-Moussa, F., Ahissou, H., Adeoti, Z., Yehouenou, B., Mamadou, A., Toukourou, F. \& Sanni, A., 2006. Etude des possibilités de contamination des aliments de rues au bénin: Cas de la ville de cotonou. $J$. Rech. Sci. Univ. Lomé (Togo), série A, 8, 149-156.

Badarou, S. \& Coppieters, Y., 2009. Intoxications alimentaires dues à l'endosulfan: Mise en place d'un système de notification et de prise en charge au bénin'. Environnement risques \& santé, 8, 133-136.

Bankole, H., Baba-Moussa, F., Agbankpe, J., Dougnon, T., Legonou, M., Toukourou, F. \& Baba-Moussa, L., 2012. Essai d'isolement de campylobacter dans la viande de volaille en république du bénin. International Journal of Biological and Chemical Sciences, 6, 1979-1986.

Barro, N., Sangaré, L., Tahita, M. C., Ouattara, C. \& Traoré, A. S., 2005. Les principaux agents du péril identifiés dans les aliments de rue et ceux des cantines et leur prévalence en milieu hospitalier. Mattrise des Procédés en vue d'améliorer la qualité et la sécurité des aliments, Utilisation des OGM, Analyse des risques en agroalimentaire. Ouagadougou, 8-11.

Belomaria, M. \& Khadmaoui, A., 2017. Etude $\mathrm{du}$ profil épidémiologique des toxiinfections alimentaires collectives dans la région du gharb chrarda bni-hssen de 2001 a 2012: Étude rétrospective.

Centre québécois, d. i., des aliments, et de santé, animale, du ministère, de l'agriculture, des pêcheries, et de l'alimentation, \& Barthe, C., 2009. Lignes directrices et normes pour l'interprétation des résultats analytiques en microbiologie alimentaire, Agriculture, pêcherie et al.,imentation, Direction $\mathrm{du}$ laboratoire d'expertises ...,49.

Chauliac, M., Bricas, N., Ategbo, E., Amoussa, W. \& Zohoun, I., 1998. L'alimentation hors du domicile des écoliers de cotonou (bénin). Cahier Santé, 8, 101Á.

Coulibaly, W. H., N'guessan, K. F., Coulibaly, I., Djè, K. M. \& Thonart, P., 2014. Les levures et les bactéries lactiques impliquées dans les bières traditionnelles à base de sorgho produites en afrique subsaharienne (synthèse bibliographique). Biotechnologie, Agronomie, Société et Environnement, 18, 209-219.

Davis, G. S., Waits, K., Nordstrom, L., Weaver, B., Aziz, M., Gauld, L., Grande, H., Bigler, R., Horwinski, J., Porter, S., Stegger, M., Johnson, J. R., Liu, C. M. \& Price, L. B., 2015. Intermingled klebsiella pneumoniae populations between retail meats and human urinary tract infections. Clinical Infectious Diseases, 61, 892-899.

Diallo, K. K., 2010. Frequence d'isolement des klebsiella au laboratoire de bacteriologie cvd du chu gabriel toure de 2002 a 2007. Thèse Pour obtenir le Grade de Docteur en Pharmacie (Diplome D'etat), Université de BamakoFaculté de médecine de pharmacie et d'odonto-stomatologie. 96

Ennadir, J., Hassikou, R., Ohmani, F., Hammamouchi, J., Bouazza, F., Qasmaoui, A., Mennane, Z., Touhami, A. O., Charof, R. \& Khedid, K., 2012. Qualité microbiologique des farines de blé consommées au maroc. Canadian journal of microbiology, 58, 145-150.

FAO 2001. Annexe 2: Application de l'analyse des risques aux programmes de contrôle de la sécurité sanitaire des 
aliments. In: Fao (ed.) Systèmes de qualité et de sécurité sanitaire des aliments. :.

FAO, 2013. Bonnes pratiques de transformation du soja en fromage. Technologies and pratices for small agricultural producers, 6.

Fayomi B., J. R., Adaye A., Laloe V., Djogbe H., Zohoun R., 1992. Toxi-infection alimentaire en milieu rural beninois. Medecine d'Afrique Noire, 39, 364-366.

Guérin, F., 2015. Infections à enterobacter cloacae complex: Résistance aux antibiotiques et traitement. Journal des Anti-infectieux, 17, 79-89.

Guiraud, J. \& Galzy, P., 1980. L'analyse microbiologique dans les industries alimentaires. Paris, France, 236 p.

Haour, A., 2018. Toxi-infections alimentaires colletcives, vue d'ensemble (exemple du maroc 2008-2017) et mise en relief sur le cas particulier de listériose. 180

Houssou, P., Vodouhè, M., Dansou, V., Todohoue, C. \& Hotegni, A., 2015. Diagnostic des technologies de transformation du soja en lait et produits dérivés au bénin. 52p.

INSAE 2013. Résultats provisoires du rapport général de la population et de l'habitat $4^{\text {ème }}$ édition. Bénin.

ISO 4833, 2003. (international organization for standardization 4833), microbiologie des aliments, méthode horizontale pour le dénombrement des microorganismes, technique de comptage des colonies à $30^{\circ} \mathrm{c}, .9 \mathrm{p}$.

ISO 6579, 2002. (international organization for standardization 6579), microbiology of food and animal feeding stuffs, horizontal method for the detection of salmonella spp, fourth edition,. $27 \mathrm{p}$.

Kaferstein, F. K. \& Abdussalam, M., 1999. La salubrité des aliments au xxie siècle/f. Käferstein et m. Abdussalam.

Malvy, D., Djossou, F. \& Bras, L., 2003. Infections et toxi-infections d'origine alimentaire et hydrique: Orientation diagnostique et conduite à tenir. EMC Pathologie professionnelle et de l'environnement, 276-284.

Michaud, A. \& Vodouhè, G., 2012. L L émergence des innovations au bénin: Cas du soja et des produits dérivés de grande consommation. FSA, CIRAD, JOLISSA. Rapport d" étude. 47p.

Monsel, G., Dauendorffer, J.-N., Aubry, A., Nebbad, B., Schneider, P., Caumes, E. \& Chosidow, O., Enterobacter aerogenes: Un nouvel agent de folliculite des jacuzzis? Annales de Dermatologie et de Vénéréologie, 2016. : Elsevier, S334.

NF EN ISO 6888-1/A1 (V 08-014-1/A1), 2004. (norme française en iso 6888-1/ amendement 1 - indice de classement : V 08-014-1/a1), microbiologie des aliments, méthode horizontale pour le dénombrement des staphylocoques à coagulase positive (staphylococcus aureus et autres espèces), partie 1 : Technique utilisant le milieu gélosé de baird-parker, amendement 1 : Inclusion des données de fidélité,. $13 \mathrm{p}$.

NF ISO 4832 (V08-060), 2009. (norme française - indice de classement : V 08060), microbiologie des aliments, dénombrement des coliformes thermotolérants par comptage des colonies obtenues à $44^{\circ} \mathrm{c}, .10 \mathrm{p}$.

NF ISO 4832 (V 08-015), 2006. (norme française iso 4832 - indice de classement : V 08-015), microbiologie des aliments. Méthode horizontale pour le dénombrement des coliformes, méthode par comptage des colonies obtenues à $37^{\circ} \mathrm{c}, .6 \mathrm{p}$.

NF ISO 21527-2, 2008. (norme française iso 21527-2), microbiologie des aliments, méthode horizontale pour le dénombrement des levures et moisissures, partie 2 : Technique par comptage des colonies dans les produits 
à activité d'eau inférieure ou égale à 0,95, (indice de classement : V 08-0402),. $9 \mathrm{p}$.

NF V08-061, 2009. Dénombrement en anaérobiose des bactéries sulfitoréductrices par comptage des colonies à $46^{\circ} \mathrm{c} .17 \mathrm{p}$.

Nguyen, T. T.,2015.Contribution à l'utilisation du lactulose comme additif fonctionnel dans la fabrication de pain riche en son de blé. Univerité de LAVAL. Mémoire de maîtrise, 118.

Rousseaux, S., Grangeteau, C., Gerhards, D., von Wallbrunn, C., Alexandre, H. \& Guilloux-Benatier, M., 2017. Origines des levures saccharomyces et nonsaccharomyces présentes dans le moût : Vignoble ou cuverie? Revue des Oenologues, 28-31.

Saoud, M. Z., Rhatous, M., El Mire, S., Lyagoubi, M. \& Aoufi, S., 2017. Saccharomyces cerevisiae, une levure de plus en plus impliquée dans les infections urinaires: À propos de 3 cas. Journal de Mycologie Médicale, 27, e31-e32.

Sossa-Minou, P. C., Sessou, P. \& Azokpota, P., 2018. Evaluation de la qualité microbiologique des aliments solides de grande consommation vendus en restauration collective sur les campus de l'université d'abomey-calavi. incomplet!

Tanouti, A., 2016. Microorganismes pathogènes portés par les aliments:
Classification, épidémiologie et moyens de prévention. $155 \mathrm{p}$.

Tingbe, V. F. A., Azonhe, T. H., Yemadje, A. \& Vido, A. A., 2018. Consommation de boissons desalterantes et risques sanitaires dans les colleges de la ville d'abomey (republique du benin). European Scientific Journal, ESJ, 14, 251.

Umba, J., Masimango, T., Kashala, J., Kusika, C. \& Musay, P., 2018. Analyse de la qualité microbiologique du pain commercialisé et consommé en l'état à kinshasa (rd congo). Journal of Animal \& Plant Sciences, 38, 6244-6256.

Union Européenne, 2005. Commission regulation (ec) no 2073/2005 of 15 november 2005 on microbiological criteria for foodstuffs. Journal the European Union.

WHO 2004. Microbial fact sheets. Guidelines for drinking-water quality. Third edition ed. : World Health Organization.

WHO 2015a. Maladies d'origine alimentaire: Près d'un tiers des décès surviennent chez les enfants de moins de 5 ans. World Health Organization: WHO. : World Health Organization: WHO.

WHO, 2015b. Who estimates of the global burden of foodborne diseases: Foodborne disease burden epidemiology reference group 20072015, World Health Organization

\section{How to cite this article:}

Djogbe A. A. M., C. K. C. Tchekessi, P. Sachi, C. Degbey, R. Bleoussi, J. Banon, K. Assogba, E. M. Ouendo and Bokossa Yaou I. 2019. Microbiological Characterization of Salty Bread, Soy Cheese and Three Yogurt Varieties Sold in the Streets of Benin. Int.J.Curr.Microbiol.App.Sci. 8(08): 2201-2216. doi: https://doi.org/10.20546/ijcmas.2019.808.255 